\title{
Obtención de la eficiencia óptica de un disco parabólico mediante método SHOT modificado
}

\section{Obtaining the parabolic dish optical efficiency using a modified SHOT method}

Presentación: 06/10/2020 y 07/10/2020

\section{Doctorando:}

\section{Dario Panaroni}

Energías Alternativas, Tecnología y Desarrollo Sustentable; Universidad Tecnológica Nacional; Facultad Regional La Plata Argentina - Comisión de Investigaciones Científicas de la Provincia de Buenos Aires - Argentina.

dario.panaroni@gmail.com

\section{Directora:}

\section{Ana Castro Luna}

\section{Co-director:}

\section{Luis Martorelli}

\section{Resumen}

La energía solar térmica concentrada es un conjunto de tecnologías capaces de obtener energía térmica de alta temperatura a partir de los rayos solares. Existen diversas formas de lograr dicho fin, pero todas ellas tienen en común dos componentes: un espejo que concentra los rayos solares y un receptor que es capaz de transformar esos rayos en energía térmica. Una característica muy importante de estos sistemas es la eficiencia óptica, pudiendo evaluarla mediante diferentes metodologías. En el presente estudio se realiza el análisis de un concentrador tipo disco parabólico para obtener su eficiencia óptica utilizando un método SHOT modificado. Luego ese resultado es comparado con aquellos obtenidos con anterioridad utilizando la metodología de calorímetro de masa constante.

Palabras clave: Energía Solar Térmica Concentrada, Energía Solar, Eficiencia Óptica.

\begin{abstract}
The concentrated solar power is a set of technologies capable of obtaining high-temperature thermal energy from solar rays. There are several ways to achieve this, but they all have two components in common: a mirror that concentrates the sun's rays and a receiver that is capable of transforming those rays into thermal energy. A very important characteristic of these systems is optical efficiency, which can be evaluated using different methodologies. In the present study, the analysis of a parabolic disk type concentrator is performed to obtain its optical efficiency using a modified SHOT method. This result is then compared with those obtained previously using the constant mass calorimeter methodology.
\end{abstract}

Keywords: Concentrated Thermal Solar Energy, Solar Energy, Optical Efficiency. 


\section{Introducción}

Los sistemas solares térmicos de concentración son aquellos capaces de concentrar los rayos solares incidentes en un área de gran tamaño para concentrarlos en una zona mucho menor, logrando aumentar la intensidad lumínica y su exergía. Este principio permite aprovechar el potencial energético que proporciona el sol para producir su conversión a energía eléctrica mediante transformaciones. Puede dividirse dos componentes principales, los cuales son combinados de diversas formas: Los concentradores ópticos encargados de recoger y concentrar los rayos solares y los receptores capaces de transformar esa energía lumínica en energía térmica.

Los colectores tipo disco parabólicos están constituidos por una superficie reflectante con una geometría parabólica de revolución la cual produce una concentración focal de geometría radial, generando una gran intensidad energética aprovechable mediante la utilización de una máquina térmica (Gordon 2013). Esta energía térmica de alta temperatura puede aplicarse en un ciclo termodinámico capaz de transformarla en energía mecánica. Existen diversos ciclos utilizados para dicho fin, pudiéndose mencionar el ciclo Rankine, Rankine orgánico, ciclo Brayton, y ciclo Stirling (Duffie and Beckman 2013). Además, esta energía térmica también podría ser utilizada por un conversor termoeléctrico como los de efecto Seebeck o el Conversor Termoeléctrico de Metal Alcalino (AMTEC por sus siglas en inglés) (Gordon 2013; Tanaka 2010).

Estos sistemas pueden ser evaluados para obtener su eficiencia óptica $\left(\eta_{\text {op }}\right)$ que puede ser definida como (Lovegrove and Stein 2012):

$$
\eta_{\text {op }}=P_{\text {rec }} / P_{\text {con }}
$$

Donde $\mathrm{P}_{\text {rec }}$ es la potencia lumínica en el área concentrada y $\mathrm{P}_{\text {con }}$ es la potencia lumínica que impacta al concentrador. Este valor es de suma utilidad puesto indica cuanta energía es capaz de concentrar dispositivo para un potencial solar dado, pudiendo hacer predicciones de rendimiento anuales utilizando modelos meteorológicos.

Para obtener este valor existen diversas metodologías tales como la prueba Hartmann clásica (Malacara 2006) y la Scanning Hartmann Optical Test (SHOT) (Wendelin, Jorgensen, and Wood 1991). La primera utiliza una máscara con orificios que se encuentra ubicada frente al concentrador por delante de su radio de curvatura, también se coloca una fuente lumínica radial detrás del radio de curvatura. Los rayos de luz emitidos por la fuente serán reflejados por el espejo concentrador para luego pasar por los orificios de la máscara pudiendo llegar así al punto conjugado del espejo. Los rayos que no fueran reflejados de forma correcta, producto de defectos de curvatura en el espejo, serán bloqueados por la máscara.

El método SHOT utiliza un láser como fuente puntual luminosa en vez de una fuente radial, y una placa fotográfica en vez de una máscara. En esta metodología se hace un barrido por puntos discretos del concentrador, mapeando automáticamente la superficie y dejando registro en la placa fotográfica.

En el presente trabajo se evalúa un concentrador tipo disco parabólico con diámetro de 1,5 m, área colectora de $1,77 \mathrm{~m}^{2}$ y distancia focal de $0,87 \mathrm{~m}$ (Figura 1). La superficie del concentrador está compuesta por facetas de triángulos equiláteros planos de 7,83 cm de lado, siendo que cada uno de éstos fue alineado con láser de forma tal que los rayos que incidan de forma perpendicular al plano de apertura sean reflejados a una misma área focal.

En un estudio anterior (Panaroni et al. 2019) el mismo concentrador fue evaluado para obtener su eficiencia óptica mediante la utilización de un calorímetro de masa constante, obteniéndose $\eta_{\mathrm{op}}=0,71 \pm 0,02$. El presente estudio utiliza una metodología SHOT, ampliamente descrita en la bibliografía, para validar el método anteriormente mencionado. 


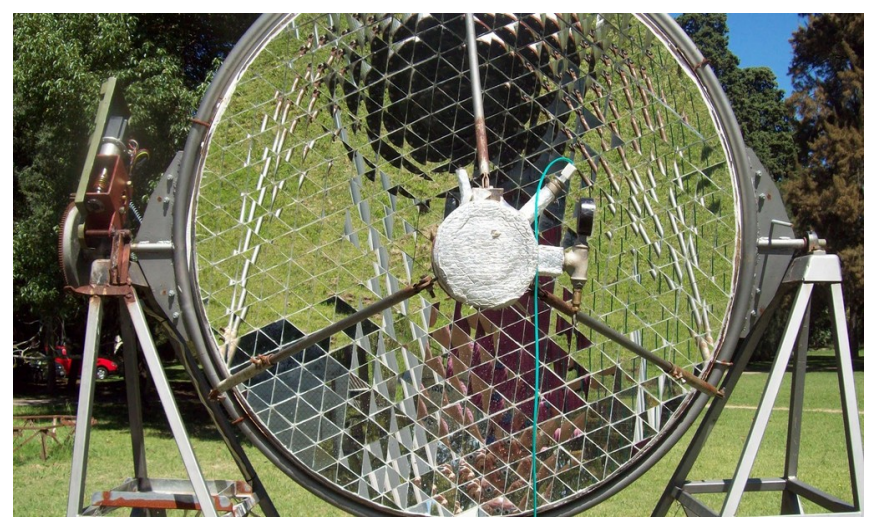

Figura 1. Colector tipo disco parabólico analizado.

\section{Desarrollo}

Para la obtención de $\eta_{\mathrm{op}}$ se realiza una prueba SHOT modificada debido a las particularidades de la superficie óptica del concentrador, que fue confeccionado con espejos planos. Dicha modificación consiste en ubicar el objeto luminoso en el infinito óptico en vez de situarse delante del radio de curvatura del concentrador. Para tal fin se posiciona un láser autonivelante a 6 metros de altura. El concentrador es colocado con su plano de apertura perpendicular al haz incidente. Mediante una plataforma mecánica fue posible desplazar el láser en coordenadas $\mathrm{x}, \mathrm{y}$ pudiendo incidir en los centros de los triángulos deseados para realizar su medición.

El haz láser impacta el espejo y es reflejado hacia un objetivo ubicado a una distancia focal sobre el ápice del concentrador, iluminando un punto sobre el mismo. El objetivo cuenta con una cuadrícula graduada permitiendo medir la posición de dicho impacto en coordenadas x,y (Figura 2).

a)

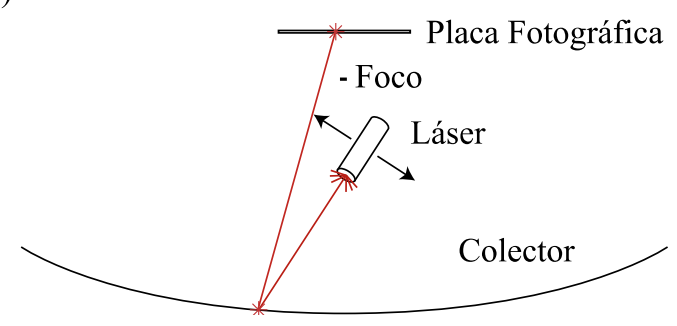

b)

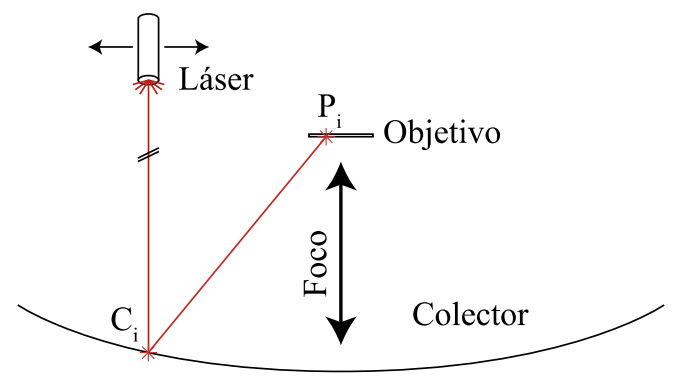

Figura 2. a) Esquema SHOT clásico. b)Esquema del método SHOT modificado.

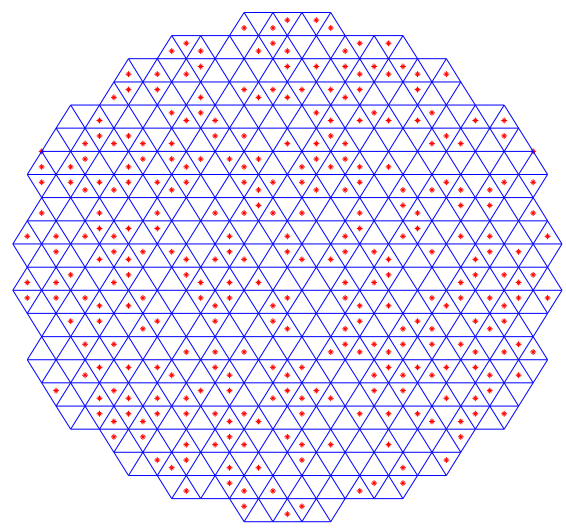


Figura 3. Vista superior del concentrador, se observa la matriz de facetas triangulares donde son marcadas en un asterisco rojo aquellos espejos que fueron evaluados.

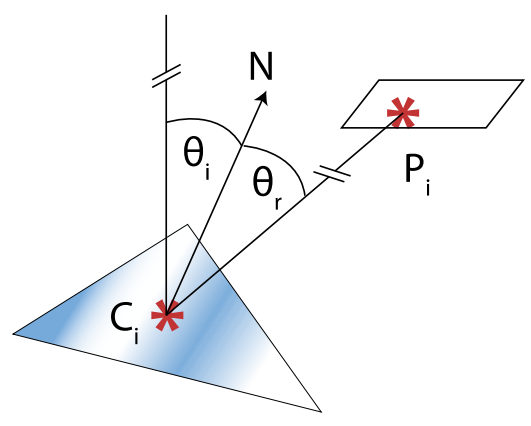

Figura 4. Ángulo de incidencia $\theta_{\mathrm{i}} \mathrm{y}$ reflexión $\theta_{\mathrm{r}}$ sobre la superficie de una faceta triangular, conjuntamente con los rayos incidente y reflejado, en medio de ellos el vector normal.

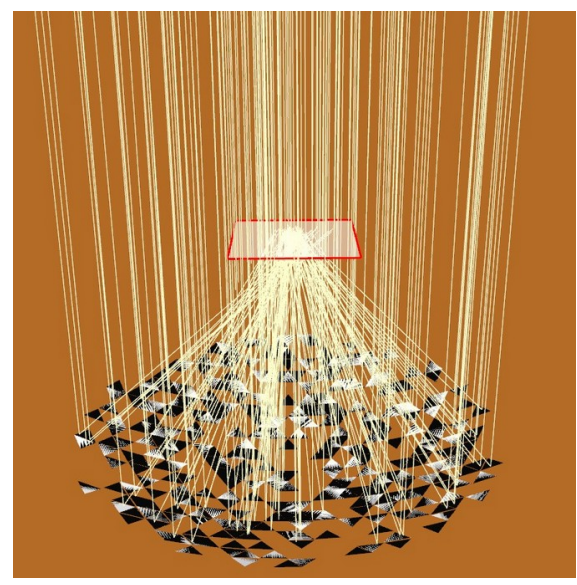

Figura 5. Simulación Monte Carlo realizada en Tonatiuh Raytracer. Se observa el modelo CAD compuesto por los triángulos muestreados.

Se ensayaron 309 facetas espejadas del concentrador, escogidas de forma aleatoria, conformando una porción representativa como se observa en la Figura 3. Para cada espejo analizado fue capaz de obtenerse una coordenada $\mathrm{Ci}(\mathrm{x}, \mathrm{y}, \mathrm{z})$ del centro del espejo y una coordenada $\mathrm{Pi}(\mathrm{x}, \mathrm{y}, \mathrm{z})$ del impacto en el objetivo. Utilizando el conjunto de datos Pi puede obtenerse el factor de intercepción $(\gamma)$ sobre un área dada. El mismo es definido como:

$$
\gamma=\text { Rayos impactados } / \text { Rayos totales }
$$

Donde los rayos impactados son aquellos que lo hicieron dentro del área central de un círculo de $0.09 \mathrm{~m}$ de diámetro, emulando la ventana del calorímetro. Además de esto la $\eta_{\mathrm{op}}$ puede escribirse como (Kalogirou 2009):

$$
\eta_{\text {opt }}=\gamma \rho
$$

Donde $\rho$ es la reflectancia de los espejos, considerados para este caso $\rho=0,950$ (Jamali 2019). Si bien este valor es una buena aproximación, no considera la superficie triangular completa de los espejos siendo ésta de un tamaño similar al área de impacto, por esto es necesario un análisis más riguroso.

Considerándose cada faceta triangular como un espejo plano y siendo que el ángulo de incidencia es igual al ángulo de reflexión como se observa en la Figura 4, puede obtenerse un vector normal a la superficie de cada espejo medido utilizando el conjunto de puntos obtenidos experimentalmente. 
Con el conjunto de vectores normales puede construirse un modelo CAD de los espejos para luego utilizarlo en el programa Tonatiuh Ray Tracer (Blanco, Amieva, and Mancillas 2005) donde se realiza una simulación por método Monte Carlo (Figura 5), obteniéndose un mapa de intensidad en la zona focal.

La simulación utiliza una distribución de Buie para los rayos solares, considerada como válida para dicho propósito (Buie, Monger, and Dey 2003). La reflectividad de los espejos fue ingresada como $\rho=0,950$ y los errores cuadráticos medios de pendiente en 0 por considerarse planos y de buena calidad.

\section{Resultados}

Utilizando como una primera aproximación el impacto del láser sobre el objetivo, se obtuvo un factor de intecepción de $\gamma=0,744$ y $\eta_{\mathrm{op}}=0,703$ (Figura 6). Si bien este valor no llega a la precisión deseada, puede servir como un primer acercamiento al resultado, brindando además una confirmación del mismo.

De la simulación por método Monte Carlo es analizado el mapa de intensidad obtenido en el área que ocupa la ventana del calorímetro (Figura 7). La radiación total impactada en el colector fue de 791,6 W mientras que dentro del área central de 0,09 m de diámetro fue de 553,1 W. Utilizando la equación (1) puede obtenerse $\eta$ op= 0.689.

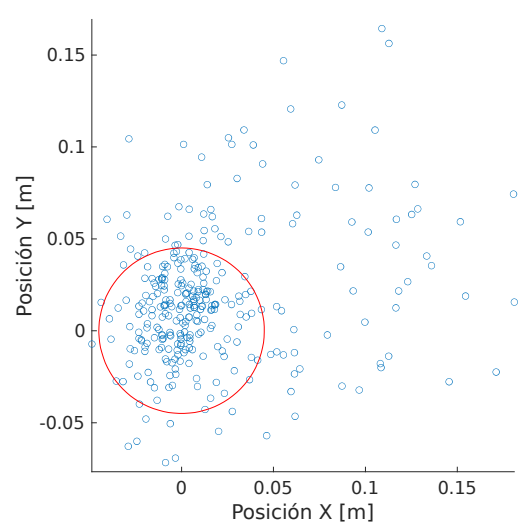

Figura 6. Puntos Pi obtenidos sobre el objetivo en círculos azules. El círculo rojo representa el área de 0,09m de diámetro por donde ingresaban los rayos al calorímetro.

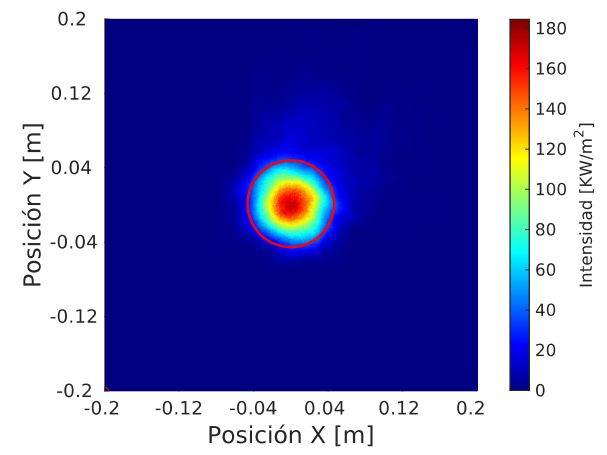

Figura 7. Mapa de intensidad resultado de la simulación Monte Carlo en el target. En círculo rojo el área central de 0,09m de diámetro. 


\section{Conclusiones}

Se ha presentado el método SHOT modificado el cual utiliza una fuente lumínica ubicada en el infinito óptico mientras se observan los impactos de sus reflejos en el foco, teniendo un comportamiento óptico equivalente al método SHOT original. Con los resultados de dicha metodología se obtiene un modelo computacional CAD con el que se realiza una simulación por método Monte Carlo de la que se obtiene un mapa de intensidad.

Mediante el método SHOT modificado se obtuvieron una $\eta_{\mathrm{op}}=0.689$ valor similar al obtenido por el método del calorímetro de masa constante que fue de $\eta_{\mathrm{op}}=0,71 \pm 0,02$. Esta coincidencia de ambos valores sirve como validación del método de calorímetro constante.

\section{Referencias}

Blanco, Manuel J., Juana M. Amieva, and Azael Mancillas. 2005. "The Tonatiuh Software Development Project: An Open Source Approach to the Simulation of Solar Concentrating Systems." In Computers and Information in Engineering, ASME, 157-64. http://proceedings.asmedigitalcollection.asme.org/proceeding.aspx?articleid=1580262 (November 24, 2017).

Buie, D., A. G. Monger, and C. J. Dey. 2003. “Sunshape Distributions for Terrestrial Solar Simulations.” Solar Energy 74(2): 113-22.

Duffie, John A, and William A Beckman. 2013. 3 Solar Engineering of Thermal Processes. Wiley New York.

Gordon, J M. 2013. Solar Energy: The State of the Art. Taylor \& Francis.

Jamali, Hamzeh. 2019. "Investigation and Review of Mirrors Reflectance in Parabolic Trough Solar Collectors (PTSCs).” Energy Reports 5: 145-58.

Kalogirou, Soteris A. 2009. "Solar Energy Collectors.” In Solar Energy Engineering, Elsevier, 121-217. https://linkinghub.elsevier.com/retrieve/pii/B9780123745019000030 (August 28, 2020).

Lovegrove, Keith, and Wes Stein. 2012. Concentrating Solar Power Technology: Principles, Developments and Applications. eds. K Lovegrove and W Stein. Woodhead Publishing. https://www.elsevier.com/books/concentratingsolar-power-technology/lovegrove/978-1-84569-769-3 (November 24, 2017).

Malacara, Danial. 2006. Optical Shop Testing: Third Edition Optical Shop Testing: Third Edition.

Panaroni, D, M Mujica, L Martorelli, and A M Castro Luna. 2019. "Determinación de La Eficiencia Óptica de Un Colector Tipo Disco Parabólico Mediante Calorimetría.” Ingenio Tecnológico 1.

http://ingenio.frlp.utn.edu.ar/index.php/ingenio/article/view/11.

Tanaka, Kotaro. 2010. "Concept Design of Solar Thermal Receiver Using Alkali Metal Thermal to Electric Converter (AMTEC).” Current Applied Physics 10(2): S254--S256.

Wendelin, T J, G J Jorgensen, and R L Wood. 1991. "SHOT: A Method for Characterizing the Surface Figure and Optical Performance of Point Focus Solar Concentrators.” In Proceedings of the ASME-JSES-JSME International Solar Energy Conference, , 555-60. 